\title{
Trading wind generation from short-term probabilistic forecasts of wind power
}

\author{
Pinson, Pierre; Chevallier, Christophe; Kariniotakis, Georges
}

Published in:

IEEE Transactions on Power Systems

Link to article, DOI:

10.1109/TPWRS.2007.901117

Publication date:

2007

Document Version

Publisher's PDF, also known as Version of record

Link back to DTU Orbit

Citation (APA):

Pinson, P., Chevallier, C., \& Kariniotakis, G. (2007). Trading wind generation from short-term probabilistic forecasts of wind power. IEEE Transactions on Power Systems, 22(3), 1148-1156.

https://doi.org/10.1109/TPWRS.2007.901117

\section{General rights}

Copyright and moral rights for the publications made accessible in the public portal are retained by the authors and/or other copyright owners and it is a condition of accessing publications that users recognise and abide by the legal requirements associated with these rights.

- Users may download and print one copy of any publication from the public portal for the purpose of private study or research.

- You may not further distribute the material or use it for any profit-making activity or commercial gain

- You may freely distribute the URL identifying the publication in the public portal

If you believe that this document breaches copyright please contact us providing details, and we will remove access to the work immediately and investigate your claim. 


\title{
Trading Wind Generation From Short-Term Probabilistic Forecasts of Wind Power
}

\author{
Pierre Pinson, Christophe Chevallier, and George N. Kariniotakis, Member, IEEE
}

\begin{abstract}
Due to the fluctuating nature of the wind resource, a wind power producer participating in a liberalized electricity market is subject to penalties related to regulation costs. Accurate forecasts of wind generation are therefore paramount for reducing such penalties and thus maximizing revenue. Despite the fact that increasing accuracy in spot forecasts may reduce penalties, this paper shows that, if such forecasts are accompanied with information on their uncertainty, i.e., in the form of predictive distributions, then this can be the basis for defining advanced strategies for market participation. Such strategies permit to further increase revenues and thus enhance competitiveness of wind generation compared to other forms of dispatchable generation. This paper formulates a general methodology for deriving optimal bidding strategies based on probabilistic forecasts of wind generation, as well as on modeling of the sensitivity a wind power producer may have to regulation costs. The benefits resulting from the application of these strategies are clearly demonstrated on the test case of the participation of a multi-MW wind farm in the Dutch electricity market over a year.
\end{abstract}

Index Terms-Decision-making, energy markets, forecasting, uncertainty, wind energy.

\section{INTRODUCTION}

W ITH the liberalization of electricity markets, wind energy producers have the possibility to dispatch their production through electricity pools, rather than having recourse to bilateral contracts. The main characteristic of these markets is that one has to propose bids in advance, and is then charged for any imbalance, defined hereafter as the deviation between the actual production and the energy bid. Owing to the problem of predictability, the market value of wind energy is reduced by the cost for regulation [1].

The participation of wind energy producers in electricity pools is a decision-making problem: the characteristics of regulation prices may encourage them to be more strategic in their way of bidding on the day-ahead market in order to restrain these regulation costs [2]. The definition of participation strategies calls for forecasts of future wind generation, typically for the next 24-72 h, which may be produced from either reference or advanced methods. While forecast accuracy is the main focus of forecasters, forecasts' users are concerned with

Manuscript received October 9, 2006; revised February 14, 2007. This work was supported in part by the European Commission under the ANEMOS Project (ENK5-CT2002-00665). Paper no. TPWRS-00709-2006.

P. Pinson is with the Informatics and Mathematical Modeling Department, Technical University of Denmark, 2800 Kgs. Lyngby, Denmark (e-mail: pp@imm.dtu.dk).

C. Chevallier and G. N. Kariniotakis are with the Center for Energy and Processes, Ecole des Mines de Paris, 06904 Sophia-Antipolis, France (e-mail: christophe.chevallier@ensmp.fr; georges.kariniotakis@ensmp.fr).

Digital Object Identifier 10.1109/TPWRS.2007.901117 the maximization of the benefits (economical or not) resulting from the use of predictions. This is what is commonly referred to as the forecast value [3]. The present paper contributes to the discussion on the value of wind power forecasts in a market environment, by showing how associated uncertainty estimates increase that value.

So far, several studies concerning the participation of wind energy in electricity markets have been carried out, considering different market mechanisms and various prediction methodologies. Some of them focus on the use of point predictions and relate the accuracy of the forecasts to the resulting regulation costs [4], [5]. In contrast, methods described in [6] and [7] integrate information on prediction uncertainty for taking advantage of the asymmetry of imbalance prices, while [8] and [9] describe participation strategies based on optimal quantile forecasts of wind generation. Finally, [10] proposes a complete methodology that accounts for the uncertainty in both wind power predictions and imbalance prices by using scenarios of power production and imbalances prices, consequently used in a stochastic optimization problem. Our aim in the present paper is to compare the market value of different wind power forecasting methods and their associated bidding strategies. Two rival approaches are considered, i.e., point forecasting and probabilistic forecasting methods. It is shown how the latter ones outperform the former ones when used in conjunction with appropriate bidding strategies. Such optimal bidding strategies are described. They use the utility theory in order to build a model of the sensitivity of the market participant to regulation costs. Both probabilistic forecasts and this model are consequently integrated in a suitable decision-making process in a stochastic optimization framework.

Initially, the main characteristics of electricity markets are reviewed. It is explained how wind energy may be penalized in comparison to dispatchable generation. Then, the assumptions for the present study are given and the problem is formulated in a general manner, so that the application of the proposed bidding strategies can be extended to other cases. The methodology for the definition of optimal bidding strategies follows. Finally, the participation of a real multi-MW wind farm in the Dutch electricity pool is simulated over a year, illustrating the benefits of the introduced methodology for deriving optimal bids from probabilistic predictions of wind generation in comparison to bids based on point forecasts only.

\section{TRADING Wind GENERATION IN ELECTRICITY MARKETS}

\section{A. Description of Electricity Markets}

At any time, the total amount of produced electricity must meet consumption. Electricity markets may be considered as an 
alternative solution to power units scheduling, since they guarantee a cost-effective match between supply and demand bids. Each electricity pool has its own rules, defining the way electricity is to be sold or purchased, how the prices are settled, and the obligations to which the participants are committed. In order to stimulate the development of renewables, some pools have special rules supporting wind generation, such as guaranteed prices or no program-responsibility. An overview of European electricity pools is given in [11] while the regulatory frameworks for the integration of wind energy in some of the European countries are described in [12].

Electricity exchanges first take place in the day-ahead (spot) market, where participants (sellers and buyers) have to propose before gate closure their quantity-price bids over the following delivery period, for every unit of time hereafter referred to as program time unit (PTU). The bids are all matched through a single auction process for determining the market clearing price (also referred to as spot price) and the program of the participants for each PTU. Participants are then financially responsible for any deviation from contract. Because of the significant delay between gate closure and the beginning of the energy delivery period (up to 12-14 h), certain electricity pools also integrate intraday markets, where it is possible to take corrective actions. Gate closure on intraday markets occurs between $30 \mathrm{~min}$ and $2 \mathrm{~h}$ before time of delivery.

The regulation market, which is managed by the transmission system operator (TSO), ensures the real-time balance between generation and load. Even if the settlement mechanism for imbalance prices varies from one country to the other, the general principles remain the same: 1 ) in the case for which production perfectly matches consumption, no regulation is needed; 2 ) if the production is not sufficient to meet the load, this leads to an up-regulation situation, for which it is necessary to increase generation (or alternatively to decrease consumption); and 3) if production exceeds consumption, one faces a downregulation situation, where generation has to be reduced (or consumption to be increased). If the grid has good interconnections with neighboring systems, a part of the excess energy may be exported, but more often, generation curtailment is envisaged for lowering generation. The connections between various areas of the overall power system may also step in the regulation problem: due to a limitation of these connections, it may be necessary to dispatch both positive power in a certain area and negative power in another area. Consequently, imbalance prices are generally highly variable and hardly predictable. They should reflect the production (or curtailment) costs, include a premium for readiness, and discourage power producers to plan imbalances.

\section{B. Assumptions}

In order to simulate the participation of a wind energy producer in electricity pools or for developing advanced bidding strategies, some assumptions about the impact of wind generation on the behavior of the markets must be formulated. In addition, a thorough description of the framework in which the present study is carried out will allow us to establish to what extent the results obtained can be generalized.

It is expected that if large amounts of wind power are introduced in a power system, this would tend to lower the average spot price on the related electricity pool in the long-term [3]. Model simulations on the Nord Pool, which is a market highly penetrated by wind generation, have shown that wind power should affect the spot market also in the short-run, more precisely by producing a downward pressure on market prices [14]. However, when analyzing the Nord Pool market data over 2002 and 2003, [13] found that this trend was not statistically significant. Here, potential effects related to wind penetration in the market are neglected. Wind power producers are considered as price-takers, i.e., as economic entities that are too small to affect alone the market clearing price.

Owing to its nondispatchable and not easily predictable nature, the participation of wind generation in electricity pools compulsorily yields a certain volume of imbalances that would not occur if only conventional units were proposing bids on the market. Though, in the same way that we have made the assumption that a single wind power producer had no market power, we also assume that the bidding policy of this power producer alone cannot impact imbalance prices. Note that this assumption is implicit in the developments carried out in [8] and [9], while it is explicitly formulated in [6].

Moreover, it is assumed that wind power producers act in electricity markets as conventional producers and do not benefit from derogatory rules. This is consistent with foreseen future developments of electricity markets. In addition, it is considered that they do not make any bids for regulation or reserve power supply, or corrective actions in intraday markets. A final assumption that will be made for the case-study of the present paper (see Section IV) is that wind power producers do not apply control strategies on their production. This is because focus is given to the sole value of forecasting in a market environment. However, the proposed methodology can account for these control aspects or internal balancing possibilities used for optimally facing imbalances, depending on their magnitude. This is explained in Section III-B1, where the loss function of the wind power producer is introduced. This "no-control" assumption used for the case study is thus a restrictive assumption on the value of wind generation in electricity pools in comparison to the case for which wind is incorporated into a broader resource portfolio. Combining wind generation with conventional means or storage allows one to lower the amount of imbalances on the market. For instance, combined wind-storage systems are studied in [15], and the possibility of combining wind and hydro power generation is considered in [16].

\section{Formulation of the Problem}

For any PTU $t+k$, a market participant has to propose a level of contracted energy $E_{t+k}^{c}$. The $(t+k)$-index is used for designating a given PTU since it indeed corresponds to a lead time $t+k$ when bids are proposed at time $t$. The revenue $\mathrm{R}_{t+k}$ of a market participant proposing an amount of energy $E_{t+k}^{c}$ but actually generating $E_{t+k}^{*}$ can be formulated as

$$
\mathrm{R}_{t+k}=\pi_{t+k}^{c} E_{t+k}^{c}+T_{t+k}^{c}
$$

where $\pi_{t+k}^{c}$ is the spot price for this PTU, and $T_{t+k}^{c}$ is the imbalance cost on the regulation market. The imbalance $d_{t+k}^{*}$ is defined as

$$
d_{t+k}^{*}=E_{t+k}^{*}-E_{t+k}^{c}
$$


and consequently, $T_{t+k}^{c}$ is given by

$$
T_{t+k}^{c}= \begin{cases}\pi_{t+k}^{c,+} d_{t+k}^{*}, & d_{t+k}^{*} \geq 0 \\ \pi_{t+k}^{c,-} d_{t+k}^{*}, & d_{t+k}^{*}<0\end{cases}
$$

with $\pi_{t+k}^{c,+}$ and $\pi_{t+k}^{c,-}$ the imbalance prices for positive and negative imbalances, respectively.

Note that these two imbalance prices depend on the considered regulation mechanism. In certain cases, they simply equal a certain proportion of the market clearing price-for instance in Spain, they are such that $\pi_{t+k}^{c,+}=(1-\tau) \pi_{t+k}^{c}$ and $\pi_{t+k}^{c,-}=$ $(1+\tau) \pi_{t+k}^{c}$ [5]. More generally, these imbalance prices are asymmetric and follow a more complex function of the spot price. For some other regulation mechanisms, e.g., on the Nord Pool, they may even depend on the sign of the imbalance as a whole, so that participants who offset the system imbalance are not penalized [2], [9].

Eq. (1) can be reformulated such that the revenue $R_{t+k}$ of a participant for PTU $t+k$ results from the combination of the income from the selling of actual wind generation $E_{t+k}^{*}$ at the spot price, minus the costs for regulation, i.e.

$$
\mathrm{R}_{t+k}=\pi_{t+k}^{c} E_{t+k}^{*}-T_{t+k}^{*}
$$

where

$$
T_{t+k}^{*}=\left\{\begin{array}{ll}
\pi_{t+k}^{*,+} d_{t+k}^{*}, & d_{t+k}^{*} \geq 0 \\
-\pi_{t+k}^{*,-} d_{t+k}^{*}, & d_{t+k}^{*}<0
\end{array} .\right.
$$

$\pi_{t+k}^{*,+}$ and $\pi_{t+k}^{*,-}$, which are referred to as the regulation unit costs for positive and negative imbalances, respectively, are readily given by

$$
\begin{aligned}
& \pi_{t+k}^{*,+}=\pi_{t+k}^{c}-\pi_{t+k}^{c,+} \\
& \pi_{t+k}^{*,-}=\pi_{t+k}^{c,-}-\pi_{t+k}^{c}
\end{aligned}
$$

The formulation given by (4) has the advantage that the first component of the revenue is a "fatal" component and indeed corresponds to the income one would receive if using perfect predictions. Moreover, since the contracted energy only appears in the second component of the participant's revenue, maximizing $\mathrm{R}_{t+k}$ now translates to minimizing the costs for regulation $T_{t+k}^{*}$. In the specific case for which a market participant is not charged for his imbalance since this imbalance helps the regulation process, $T_{t+k}^{*}=0$.

Owing to the nature of regulation costs, which are mainly penalizing, the revenue can also be written in the form of a performance ratio $\gamma$. It will be used for the evaluation of the rival bidding strategies. The performance ratio $\gamma$ is calculated over a certain period of time by normalizing the actual revenue by the revenue that would be obtained if one had the possibility to use perfect forecasts. Considering an evaluation period of $N_{T}$ participation days in the electricity pool, each participation day covering $N_{\mathrm{PTU}}$ daily PTUs, $\gamma$ writes

$$
\gamma=1-\frac{\sum_{t=1}^{N_{T}} \sum_{k=1}^{N_{\mathrm{PTU}}} T_{t+k}^{*}}{\sum_{t=1}^{N_{T}} \sum_{k=1}^{N_{\mathrm{PTU}}} \pi_{t+k}^{c} E_{t+k}^{*}} .
$$

The proposed performance ratio is such that $\gamma \in(-\infty, 1]$, and it will be expressed in percent. It is obvious that for perfect prediction, $\gamma=1$ since deviations are null.

\section{Definition of AdVANCEd BIDDING StRATEgIES}

\section{A. Base-Line Strategies Based on Point Predictions}

Point prediction methods provide estimates of the expected power production of a wind farm with a forecast length of up to 2-3 days ahead and with a forecast resolution ranging from $15 \mathrm{~min}$ to $1 \mathrm{~h} \mathrm{[17].} \mathrm{For} \mathrm{a} \mathrm{given} \mathrm{look-ahead} \mathrm{time} t+k$, they give estimates of the average power output between lead times $t+k-$ 1 and $t+k$. Therefore, it appears reasonable to give a forecast of the wind energy generated during that period as the product of the average power production by the forecast resolution $t_{r}$. If denoting by $\hat{p}_{t+k \mid t}$ and $\hat{E}_{t+k \mid t}$, the power and energy forecasts, respectively, issued a time $t$ for lead time $t+k$, this writes

$$
\hat{E}_{t+k \mid t}=\hat{p}_{t+k \mid t} t_{r}
$$

For convenience, power forecasts are normalized by the nominal power $P_{n}$ of the considered wind farm, and energy forecasts by $P_{n} . t_{r}$, so that they take values in the unit interval. If point predictions are the only information one has on future wind generation, then they comprise the best bid $\tilde{E}_{t+k}^{c}$ one can propose for PTU $t+k$ as follows:

$$
\tilde{E}_{t+k}^{c}=\hat{E}_{t+k \mid t}
$$

\section{B. Optimal Bidding Strategies Based on Probabilistic Forecasts}

Instead of seeing $E_{t+k}^{*}$ as a true effect one wants to predict as accurately as possible with $\hat{E}_{t+k \mid t}$, one may have a probabilistic view of the problem, by considering that $E_{t+k}$ is a random variable and that $E_{t+k}^{*}$ is a realization of that random variable. Point predictions $\hat{E}_{t+k \mid t}$ produced in a minimum square error sense are indeed estimates of the conditional expectation $\bar{E}_{t+k}$ of the distribution of $E_{t+k}$ (conditional to the information set available at time $t$ ). Denote by $F_{t+k}^{E}$ the density function of $E_{t+k}$. Though, the expectation is only a summary statistics of what can be the realization $E_{t+k}^{*}$, and it cannot give an information on what could happen. Note that from now on, the imbalance $d_{t+k}$ is also seen as a random variable, defined as

$$
d_{t+k}=E_{t+k}-E_{t+k}^{c}
$$

such that $d_{t+k}^{*}$ denotes a realization of that random variable.

Hereafter, it is supposed that a forecast $\hat{F}_{t+k \mid t}^{E}$ of the density function of $E_{t+k}$ is available at time $t$, given, e.g., by the expert model described in [18] and [19, Ch. 4]. Probabilistic forecasts are the basis of the methodology proposed below for designing optimal bidding strategies, accompanied by the modeling of the sensitivity of the market participant to regulation costs, which are then both integrated in a suitable decision-making process.

1) Modeling the Market Participant's Utility Function: The utility assigns a degree of happiness in the form of a single numerical value to every possible outcome with which a decision-maker may be faced. Following the formulation of the participant's revenue given by (4), maximizing the participant's 
utility function directly translates to minimizing the loss function associated to regulations costs. Modeling the perceived loss for the wind power producer as a function of the amount of regulation may not be an easy task [9], [20]. Ideally, this model results from a discussion between an analyst and the wind power producer.

In a first stage, let us define the basic properties of a function $g$, which gives the loss associated to a given imbalance $d^{*}$ as follows:

$$
g: d^{*} \in[-1,1] \rightarrow g\left(d^{*}\right) \in \mathbb{R}^{+} .
$$

The imbalance $d^{*}$ is contained in the range $[-1,1]$ since we work with normalized variables. Values of $g\left(d^{*}\right)$ are always greater than or equal to 0 , following the assumption that a market participant does not expect to be rewarded for an imbalance. It may be possible to build a loss function depending on $k$ or $t$, in order to account for diurnal or seasonal trends in regulation unit costs. The loss functions used in the case study of the paper are independent of $k$, and we will study the benefits of introducing a different loss function depending on the season of the year.

The function $g$ is defined as a continuous piecewise linear function, in order to reflect different level of losses for different ranges of imbalances. It is assumed that the loss is strictly increasing when the deviation from the level of contracted energy increases. This translates to $g^{\prime}$ being an increasing function such that $g^{\prime}\left(d^{*}\right)<0$ for $d^{*}<0$ and $g^{\prime}\left(d^{*}\right)>0$ for $d^{*}>0$. Note that $g^{\prime}$ may not be defined for certain values of $d^{*}$, since $g$ is piecewise. This set of properties leads to $g$ being a convex function. Finally, $g$ must be such that $g(0)=0$, since the market participant does not have to buy or sell energy on the regulation market if there is no deviation from contract. This is also in line with the idea that the use of perfect predictions would lead to the maximum income.

A straightforward way of defining $g$ is to consider that the loss is directly given by the regulation unit costs $\pi_{t+k}^{*,+}$ and $\pi_{t+k}^{*,-}$. They then define the slope of linear functions for positive and negative values of $d$ as follows:

$$
g: d^{*} \rightarrow\left\{\begin{array}{ll}
\pi_{t+k}^{*,+} d^{*}, & d^{*} \geq 0 \\
-\pi_{t+k}^{*-} d^{*}, & d^{*}<0
\end{array} .\right.
$$

The regulation unit $\operatorname{costs} \pi_{t+k}^{*,+}$ and $\pi_{t+k}^{*,-}$ are not known when bidding at time $t$. Consequently, they have to be replaced by forecasts or estimates in (13). These forecasts can be obtained by models using as input the market clearing price [2], [21] (which is settled a few hours after gate closure). In such a case, regulation prices cannot be forecast prior to defining an optimal bid, unless using in a first stage a model for forecasting spot prices [22]. Alternatively, an estimation strategy consisting of climatology-like forecasts can already be valuable [23]. In the present paper, we will only assume that it is possible to predict a trend (on an annual or a quarterly basis) for the regulation unit costs.

Fig. 1 gives the example of two alternative loss functions. The first one, referred to as "market-based," represents the type of loss functions used in the present paper, for which the slope of the two linear parts is directly given by the regulation unit costs for positive and negative imbalances. Alternatively, one could use more "advanced" curves to represent the loss function, such as the piecewise linear function shown in Fig. 1. Such functions

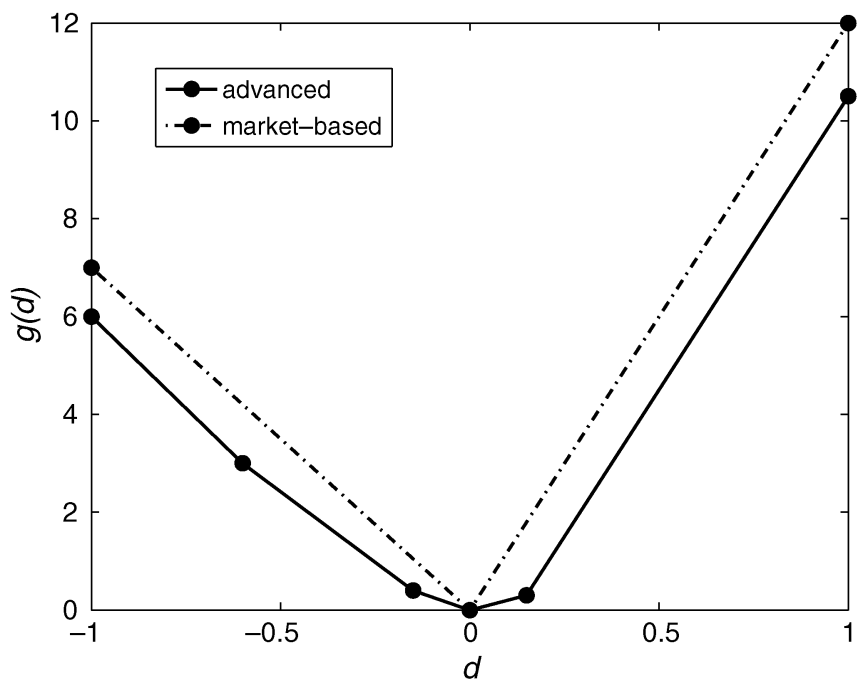

Fig. 1. Example of two different loss functions based either on the regulation unit costs only or on expertise reflecting the sensitivity of the wind power producer to the magnitude of deviations from contract.

may be more appropriate in situations for which the sensitivity of the end-user varies with the magnitude of the deviation from contract and integrate internal balancing possibilities. They can be designed in a heuristic way. The "advanced" loss function in Fig. 1 corresponds to an example where the end-user has the possibility to combine wind energy with some storage and conventional generation when trading, as envisaged in, e.g., [15] and [16]. It then permits to account for the possibility to use this storage for compensating small positive and negative imbalances (here in the range $[-0.15,0.18])$. The related slopes and ranges in the loss function depend on the charging and discharging efficiencies of the storage, as well as the chosen control strategy. Then, for larger negative imbalances (in the range $[-0.6,-0.15])$, the wind power producer has the possibility of coupling his output with conventional generation up to a certain level. The cost of this coupling defines the slope for this range of imbalances. Finally, for imbalances outside of the range $[-0.6,0.18]$, he does not have any more option for facing imbalances, and his loss is directly defined by the unit imbalance costs on the electricity pool.

2) Bidding Strategies Tailored to End-User Needs: Given the probabilistic distribution $F_{t+k}^{E}$ of wind generation for PTU $t+k$, and given the loss function $g$, a contract level can be determined in various ways. Indeed, the definition of an optimal bidding strategy, like the modeling of the loss function, depends on the sensitivity of the market participant to penalties. The market participant may want to optimize his utility on the electricity pool over a certain period of time, or alternatively to minimize the risk of large losses since the related costs might be prohibitive. These two alternative strategies are referred to as probabilistic choice (PC) and risk adverse (RA), following the terminology introduced in [24], where these decision-making paradigms have been successfully applied for power system planning. Note that the latter type of bidding strategies is at the expense of a maximum utility on the longterm though.

Maximizing the utility of the market participant over a certain period of time is equivalent to minimizing the expectation of the 
loss function $g$ for each of these PTUs. The loss expectation for PTU $t+k$ writes

$$
\mathbb{E}\left[g\left(d_{t+k}\right)\right]=\int_{0}^{1} g\left(x-E_{t+k}^{c}\right) F_{t+k}^{E}(x) d x
$$

and the optimization problem to be solved for every PTU is as follows:

$$
\tilde{E}_{t+k}^{c}=\underset{E_{t+k}^{c}}{\arg \min } \mathbb{E}\left[g\left(d_{t+k}\right)\right] .
$$

In the case where the loss function is a direct model of the regulation unit costs [cf. (13)], the optimal bid at PTU $t+k$, which thus minimizes the expectation of $T_{t+k}^{*}$, is indeed given by

$$
\tilde{E}_{t+k}^{c}=\underset{E_{t+k}^{c}}{\arg \min } \mathbb{E}\left[T_{t+k}^{*}\right]=G_{t+k}^{E}-1\left(\frac{\pi_{k}^{*,+}}{\pi_{k}^{*,+}+\pi_{k}^{*,-}}\right)
$$

where $G_{t+k}^{E}$ is the cumulative distribution function for the random variable $E_{t+k}$ (for a proof, see [8] and [9]). In practice, an estimate of $G_{t+k}^{E}$ can be derived from a predictive distribution $\hat{F}_{t+k \mid t}^{E}$. Also, following the assumption that wind generation and regulation costs are independent random variables, $\pi_{k}^{*,+}$ and $\pi_{k}^{*,-}$ can be replaced by estimates of their expectation [6], [9], for instance, given by average values over a past period. If considering more advanced loss functions, numerical optimization methods have to be envisaged for determining the optimal bid $\tilde{E}_{k}^{c}$. For the class of loss functions described in Section III-B1 (or more generally, for the class of strictly convex loss functions such that $g^{\prime}(0)=0$ ), the optimization problem (15) admits a unique minimum over the range of possible contract levels. Local optimization methods are thus sufficient for determining optimal bids. A proof of this statement is given in the Appendix.

Eq. (16) tells that the optimal bid $\tilde{E}_{t+k}^{c}$ one can make on an electricity pool for maximizing the participant's income is not given by a point forecast (which is an estimate of the wind generation expectation) but instead by a particular quantile of predictive distributions of wind generation. The proportion of this quantile is a direct function of the regulation prices. Therefore, when defining market bids from point forecasts only, it is not necessarily the most accurate point forecasting method (in a minimum square error sense) that would lead to the higher revenue on the market. This is because the criterion used for estimating the accuracy (or quality) of point predictions is not the same as the one considered for assessing their value in an operational context.

Alternatively, in meteorological situations exhibiting low predictability, a wind power producer might prefer to minimize the risk of large losses and thus use an RA strategy. Then, the optimization problem consists in finding the bid $\tilde{E}_{t+k}^{c}$ that minimizes the worst possible scenario. Such a minimax problem can be formulated as

$$
\tilde{E}_{t+k}^{c}=\underset{E_{t+k}^{c}}{\arg \min } \max _{x} g\left(x-E_{t+k}^{c}\right) F_{t+k}^{E}(x)
$$

where probabilistic distributions $F_{t+k}^{E}$ are to be replaced by predictive distributions $\hat{F}_{t+k \mid t}^{E}$. The optimization problem (17) can be solved with appropriate numerical methods.
Note that when applying a risk adverse policy, the loss function must reflect the participant aversion for losses, which is obviously not optimally represented by linear functions with slopes given by averages of regulation unit costs. In fact, it would be preferable to consider upper bounds on the value of expected imbalance costs, or alternatively to use quadratic loss functions. This is discussed in [6].

\section{Evaluation of Bidding Strategies ON AN ELECTRICITY POOL}

\section{A. Case Study}

1) Electricity Pool: The participation of a wind power producer in the Dutch electricity pool is simulated over 2002. This pool includes the day-ahead market APX, to which is associated the regulation market run by TenneT, the TSO for the Netherlands. The gate closure on APX is at 10:30 for the following day, with a PTU length of $1 \mathrm{~h}$. Market participant are paid by APX the spot price for the contracted energy, independently of their actual production. The entrance and annual fees for participating in APX are not included in the study. APX spot and TenneT regulation markets are independent, and there is no constraint on the sign or the magnitude of imbalance prices. In some situations, regulation unit $\operatorname{costs} \pi_{k}^{*,+}$ and $\pi_{k}^{*,-}$ can be negative: electricity then becomes a waste good [23]. This appears surprising but is indeed easily explainable by the so-called must-run character of some non-flexible generators [25]. Negative prices are therefore acceptable to these power suppliers since the costs of a shutdown period are sometimes much higher.

Table I gathers the average spot and regulation prices for the whole year of 2002 but also on a monthly and quarterly basis. Regulation prices related to positive imbalances tend to be higher than those related to negative ones: the ratio between their yearly averages equals 2.7 . However, this ratio is highly variable from a quarter to the other and even more from a month to the other. During the first four months, average regulation unit costs for downward regulation are even larger than the average spot prices on the day-ahead market - the market is then more penalizing with respect to deviations from contract. In parallel, the unit costs for upward regulation are very low, even negative on average for three out of these four months. The inverse situation, i.e., high prices for negative imbalances, and low prices for positive ones, occurred two months during that year, in August and December. Over 2002, regulation unit costs were indeed negative $47.42 \%$ and $19.81 \%$ of the times for the case of negative and positive deviations from contract, respectively. Such behavior of the market could conduce participants to intend imbalances. It is argued in [26] that gaming strategies on the Dutch electricity market cannot be profitable, since it would be necessary to predict at least the sign of the overall system imbalance to apply these strategies, which is hardly feasible.

2) Wind Power Producer: The participation of the operator of a 15-MW wind farm in Ireland is considered. Wind power data consist in power measurements and in a series of 48-h ahead point predictions for the year 2002, as obtained from the application of the fuzzy-neural networks (Fuzzy-NN)-based approach introduced in [27]. This is an advanced forecasting method, the performance of which is at the typical level of performance that can be found in the state-of-the-art [17], [28]. The evaluation 
TABLE I

MARKet Characteristics For 2002: Average Spot AND Regulation PRices (IN euros/MWh) ON a Monthly (M) AND Quarterly (Q) BASIS, AS Well as THE AVERAgES OVER THE YeAR (Y)

\begin{tabular}{cccccccccc}
\hline \hline Month & $\bar{\pi}_{k}^{c}(\mathrm{M})$. & $\bar{\pi}_{k}^{*,-}(\mathrm{M})$. & $\bar{\pi}_{k}^{*,+}(\mathrm{M})$. & $\bar{\pi}_{k}^{c}(\mathrm{Q})$. & $\bar{\pi}_{k}^{*,-}(\mathrm{Q})$. & $\bar{\pi}_{k}^{*,+}(\mathrm{Q})$. & $\bar{\pi}_{k}^{c}(\mathrm{Y})$. & $\bar{\pi}_{k}^{*,-}(\mathrm{Y})$. & $\bar{\pi}_{k}^{*,+}(\mathrm{Y})$. \\
\hline 1 & 14.50 & -2.10 & 18.29 & & & & & & \\
2 & 10.00 & -0.67 & 17.96 & 11.65 & 0.33 & 16.22 & & & \\
3 & 10.43 & 3.77 & 12.40 & & & & & & \\
4 & 17.92 & -6.66 & 18.49 & & & & & & \\
5 & 39.21 & 0.93 & 9.06 & 38.38 & 1.34 & 11.13 & & & \\
6 & 58.02 & 9.74 & 5.83 & & & & & & \\
7 & 48.56 & 12.97 & 2.90 & & & & & & \\
8 & 41.00 & 23.06 & -4.30 & 41.17 & 8.22 & 8.51 & & & \\
9 & 33.94 & -11.38 & 26.93 & & & & & & \\
10 & 38.25 & 9.61 & 6.38 & & & & & & \\
11 & 29.09 & -4.40 & 18.92 & 29.38 & 6.97 & 7.61 & & \\
12 & 20.81 & 15.71 & -2.47 & & & & & &
\end{tabular}

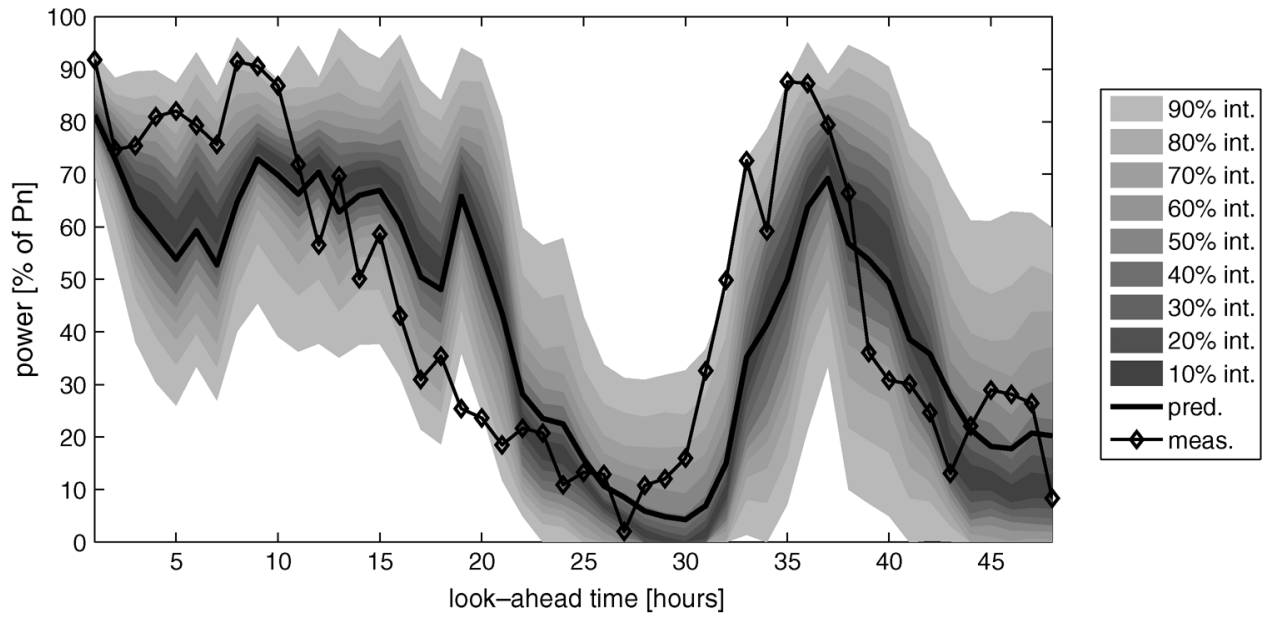

Fig. 2. Example of a 48-h ahead probabilistic forecast of normalized (by $P_{n}$ ) wind power production for a multi-MW wind farm in Ireland. Point predictions are dressed with prediction intervals whose nominal coverage range from $10 \%$ to $90 \%$, with $10 \%$ increments. A non-parametric and continuous estimate of the probabilistic distribution of wind power production for a given lead time can be readily obtained by linear interpolation through the predicted quantiles for that lead time.

methodology proposed here consists in comparing the benefits from the use of an advanced method with those that would be obtained by the use of Persistence and perfect forecasts. The Persistence prediction method consists in using the last measured power value (here at 10:00) as a prediction for all look-ahead times. The use of Persistence and perfect forecasts can be seen as the most pessimistic and optimistic cases for trading wind power. The revenues from these two methods will define an envelope in which the revenues from the use of advanced methods are expected to lie.

The PTU length on APX is of $1 \mathrm{~h}$, while it shortens to $15 \mathrm{~min}$ on the regulation market run by TenneT. Because we do not want to dilute the variations of imbalance prices, we use the TenneT PTU as the time unit for this simulation. This translates to assuming that the quantities of energy proposed on the spot market can be divided in four equal amounts of energy, for the four TenneT PTUs included in an APX PTU, sold at the same hourly spot price. A similar assumption is made for measured quantities, for comparison between contracted and actual levels of energy.

Predictive distributions are obtained by applying the adapted resampling method [18], [19, Ch. 4] to the Fuzzy-NN point forecasts. The size of error samples is set to 300 items, the number of bootstrap replications to 50, and finally five triangular fuzzy sets are used for modeling the different uncertainty regimes as a function of the level of predicted power. Interval forecasts are produced for several nominal coverage rates, from $10 \%$ to $90 \%$, with $10 \%$ increments, which is equivalent to estimating 18 quantiles of the predictive distributions of wind power for each look-ahead time. Fig. 2 depicts an example of such probabilistic forecasts in the form of a fan chart.

The wind power producer participates in the market with the aim of maximizing his revenue over 2002. As explained in Section II-B, it is assumed that the wind power producer does not apply control strategies or have internal balancing possibilities for optimally facing imbalances depending on their magnitude. Therefore, the modeling of his sensitivity to regulation costs is based on the penalties for upward and downward dispatch only (cf. "market-based" curve in Fig. 1). It is assumed that it is possible to estimate (or forecast) the annual or quarterly trends for regulation unit costs. Therefore, two PC strategies PC1 and PC2 are defined, based either on a single loss function for the whole year (PC1) or on four different loss functions for the four quarters of 2002 (PC2). In (13), estimates of regulation unit costs for upward and downward dispatch are replaced by the annual and quarterly averages given in Table I. Hence, following results rely on the assumption that one can perfectly predict trends for regulation unit costs. For every day 
TABLE II

Simulation Results Over 2002: Strategies Based on Point Predictions Only, ObTained From Persistence, the FuZZy-NN Prediction Method, and Two PC Strategies (PC1: Annual Estimates of Average Regulation Penalties—PC2: Quarterly Estimates). These Strategies are Compared to the CASe for Which Perfect PRedictions Would Be Available

\begin{tabular}{lccccc}
\hline \hline & Persistence & Fuzzy-NN & PC1 & PC2 & Perfect predictions \\
\hline Contracted energy $(\mathrm{GWh})$ & 44.37 & 45.49 & 57.23 & 62.37 & 46.41 \\
Surplus $(\mathrm{GWh})$ & 18.12 & 9.87 & 5.19 & 4.89 & 0 \\
Shortage $(\mathrm{GWh})$ & 16.08 & 8.95 & 16.03 & 20.85 & 0 \\
Down-regulation costs $\left(10^{3} €\right)$ & 195.72 & 119.99 & 55.92 & 42.61 & 0 \\
Up-regulation costs $\left(10^{3} €\right)$ & 79.59 & 52.01 & 87.15 & 61.46 & 0 \\
Total revenue $\left(10^{3} €\right)$ & 1041.38 & 1145.69 & 1173.62 & 1212.61 & 1317.69 \\
\hline Av. down-regulation unit cost $(€ / \mathrm{MWh})$ & 10.80 & 12.15 & 10.77 & 8.71 & 0 \\
Av. up-regulation unit cost $(€ / \mathrm{MWh})$ & 4.95 & 5.81 & 5.44 & 2.95 & 0 \\
Av. regulation unit cost $(€ / \mathrm{MWh})$ & 8.05 & 9.13 & 6.74 & 4.04 & 0 \\
Av. energy price $(€ / \mathrm{MWh})$ & 22.44 & 24.68 & 25.29 & 26.13 & 28.37 \\
\hline Part of imbalance $(\%$ of produced energy) & 73.69 & 40.55 & 45.72 & 55.46 & 0 \\
Performance ratio $(\gamma, \%)$ & 79.1 & 86.99 & 89.14 & 92.1 & 100 \\
\hline \hline
\end{tabular}

of the year, and for each PTU, bids are defined by solving the optimization problem (15).

\section{B. Results and Discussion}

Table II summarizes the results from the application of the various strategies, including those based on Persistence and Fuzzy-NN forecasts, as well as PC1 and PC2.

\section{Strategies Based on Point Predictions}

The prediction biases for the Persistence and the Fuzzy-NNbased methods equal $4.4 \%$ and $1.98 \%$ (of produced energy): both methods tend to slightly under-predict wind generation. The amount of energy in surplus is indeed higher than that in shortage. Therefore, the wind farm operator is more exposed to penalties for positive imbalances. This, combined with the fact that unit costs for such positive deviations from contract are larger than those for negative deviations, makes that the regulation costs associated to the energy in surplus are much higher than those related to shortage. However, the overall regulation costs are substantially reduced thanks to the use of an advanced approach instead of Persistence. The performance ratio $\gamma$ [cf. (8)] equals $79.1 \%$ if using Persistence forecasts and $86.99 \%$ if using Fuzzy-NN forecasts. In other words, regulation costs are diminished by $37.75 \%$, when preferring the advanced prediction method.

In a general manner, it is owing to their higher accuracy that advanced forecasting approaches have a greater value than reference methods. Their improvement with respect to Persistence (which corresponds to a relative error reduction) reaches high levels (up to $40 \%-60 \%$ ) for horizons further than 6-10 h ahead [29]. Therefore, this directly translates to decreasing the amount of energy subject to regulation, even if the revenue on the dayahead market is rather similar. Here, the quantity of energy subject to regulation drops from $73.6 \%$ to $40.55 \%$ (of the produced energy) if defining bids from Fuzzy-NN forecasts instead of Persistence predictions. Hence, preferring advanced prediction methods significantly decreases the financial risks that the market participant may have to face.

Over 2002, the average spot price is 29.99 euros/MWh (cf. Table I). If always bidding the same amount of energy for every PTU of the year, this would be the average price per produced
MWh one would receive from APX. However, owing to production fluctuations, this statistical average does not correspond to the maximum price per produced MWh a wind power producer can expect. Here, even if using perfect predictions, that average price per produced MWh equals 28.37 euros/MWh, which is significantly lower than the statistical average. Higher quantities of wind generation are sold when the market clearing price is low and less when this price is high. When considering Persistence or Fuzzy-NN forecasts for bidding on APX, that average price per produced MWh lowers to 22.44 and 24.68 euros/MWh, respectively, due to regulation costs. In addition, one notices that the average cost per MWh subject to regulation is not the same in both cases: when using Persistence, even if the amount of energy in imbalance is much higher over the year, the average regulation unit cost is significantly lower ( 8.05 instead of 9.13 euros/MWh).

1) Strategies Based on Probabilistic Forecasts: Even with bidding strategies defined with annual averages of regulation unit costs, the revenue of the market participant is higher than if applying strategies based on point forecasts only. While the performance ratio $\gamma$ equals $86.99 \%$ when using Fuzzy-NN forecasts, this ratio reaches $89.14 \%$ with the strategy PC1. Because of the significant variations of regulation unit prices, defining different loss functions depending on the period of the year allows one to further increase the resulting income: the performance ratio reaches $92.1 \%$ when applying the strategy PC2. This indeed means that the regulation costs over 2002 are reduced by $39 \%$ when considering the PC2 strategy instead of that based on Fuzzy-NN predictions only. Consequently, the more one can integrate information on the regulation unit costs behavior, by increasing the resolution of forecasts of these costs, the more the revenue from participation in the electricity market would increase. Though, one must remember that it is particularly difficult to estimate regulation prices with a high forecast resolution [2], [26]). In addition, the proposed strategies follow from the assumption that wind generation has no influence on regulation unit costs in the short-run and thus that the corresponding random variables can be considered as independent. This may not be true for markets significantly penetrated by wind.

The revenue of the wind power producer is not increased by reducing the amount of energy in imbalance. It is actually the 
inverse: while this amount is equal to $40.55 \%$ of the contracted energy for the bidding strategy based on Fuzzy-NN forecasts, it rises to $45.72 \%$ and $55.46 \%$ for the cases of strategies PC1 and PC2. Further integrating information on the regulation market's behavior would lead to an orientation of the imbalances. Since on average the unit costs for positive imbalances are higher than those for negative imbalances, it is preferable to propose quantities of energy that are more possibly subject to shortage than to surplus regulation. This is preferable for both the wind power producer and the TSO, since the definition of imbalance penalties also directly reflects the sensitivity of the TSO to the balance of the system. Therefore, for other electricity markets with different behaviors, or if a given regulation market behavior evolves, it can be accounted for by modifying the loss function $g$.

Going from bidding strategies based on Fuzzy-NN forecasts only to PC strategies PC1 and PC2, the costs supported by the wind power producer over 2002 for upward and downward regulation are completely different. With PC strategies, the costs for up-regulation are slightly higher than those for down-regulation, even though the quantities of energy in shortage are much higher. In addition, the cost of a regulated MWh lowers when considering optimal bidding strategies, whatever the sign of needed regulation. On average, this cost per MWh subject to regulation is divided by more than two when applying the bidding strategy PC2 (equal to 4.04 euros/MWh) in comparison to that based on Fuzzy-NN forecasts. In this simulation, the average cost of regulation per produced $\mathrm{MWh}$, which is defined as the difference between the average energy price obtained by applying a given bidding strategy and by using perfect predictions, ranges from 2.24 euros/MWh for $\mathrm{PC} 2$ to 5.93 euros/MWh for the Persistence-forecast-based policy. These numbers cannot be generalized for other years on the Dutch market or for other electricity pools, but since all electricity markets have a similar structure, applying optimal bidding strategies is also expected to result in a diminution of regulation costs per produced $\mathrm{MWh}$.

\section{CONCLusions}

Wind power forecasting has an interest for wind power producers participating in electricity pools. However, because wind power forecasts contain an inherent part of uncertainty, and since their level of accuracy is not expected to be dramatically improved in the near future, there will always be regulation costs induced by forecasting errors. In this paper, it has been shown that integrating an uncertainty information in the decision-making process could considerably increase the market value of wind power forecasts. For that purpose, a generic methodology for designing optimal participation strategies has been described, which takes into account the forecast uncertainty in the form of predictive distributions and a model of the market participant's sensitivity to regulation costs. This methodology is flexible in the sense that it can be tailored to the specific needs of the market participant, ideally following an exchange between him and an analyst.

Simulation results have been presented on the case study of the participation of a multi-MW wind farm in the Dutch electricity pool, with revenue-maximization strategies. It has been assumed that it would be possible to estimate trends for regulation unit costs, on a annual or quarterly basis. It would be of particular interest to further investigate on that topic in order to see to what extent it is possible to model and forecast the regulation penalties. Moreover, the possibility to apply different bidding strategies depending on the expected level of uncertainty has not been discussed. Considering prediction risk indices such as those introduced in [19, Ch. 5] (and also risk indices related to expected regulation costs) may be a possibility for making a choice between the application of either PC or RA strategies. This would result in avoiding excessive regulation costs in situations exhibiting low predictability.

The introduced methodology is suitable for the case of the participation of a wind power producer in a day-ahead electricity market. It can be extended in the future (in a stochastic programming framework) to other decision-making processes related to the management or trading of wind power, for the participation in several markets with various gate closures, for the coupling of wind with storage or conventional generation, etc. All management and trading problems involving wind generation prove to be optimization under uncertainty problems.

\section{APPENDIX}

In this Appendix is given the proof of the uniqueness of the solution of the optimization problem (15), provided that $g$ has the properties described in Section III-B1. The piecewise linear function $g$ can be expressed as

$$
g(d)=\sum_{i=1}^{n}\left(c_{i} d+a_{i}\right) \mathbf{1}_{d \in\left[l_{i-1}, l_{i}\right]}+a_{n+1} \mathbf{1}_{d=l_{n}}
$$

where $l_{0}=-1, l_{n}=1$. Also, there exists $m, 0<m<n$, such that $l_{m}=0$, with $c_{i}<0$ for $i \leq m, c_{i}>0$ for $i>m$, and $c_{i}<c_{i+1}, \forall i$. Finally, the continuity of $g$ imposes restrictions on the $a_{i}$ coefficients.

The loss expectation for PTU $t+k$ is given in (14). The derivative of $\mathbb{E}\left[g\left(d_{t+k}\right)\right]$ with respect to $E_{t+k}^{c}$, denoted by $h\left(E_{t+k}^{c}\right)$, writes

$$
h\left(E_{t+k}^{c}\right)=\frac{\partial}{\partial E_{t+k}^{c}} \int_{0}^{1} g\left(x-E_{t+k}^{c}\right) F_{t+k}^{E}(x) d x .
$$

By using the Leibniz rule for derivation under the integral sign, (19) becomes

$$
h\left(E_{t+k}^{c}\right)=\int_{0}^{1} F_{t+k}^{E}(x) \frac{\partial}{\partial E_{t+k}^{c}} g\left(x-E_{t+k}^{c}\right) d x
$$

which yields

$$
h\left(E_{t+k}^{c}\right)=-\int_{0}^{1} g^{\prime}\left(x-E_{t+k}^{c}\right) F_{t+k}^{E}(x) d x .
$$

From the definition of $g$, and since $F_{t+k}^{E}$ is positive, it is clear that $h(0)<0$ and $h(1)>0$. In addition, the above equation can be rewritten as

$$
h\left(E_{t+k}^{c}\right)=\sum_{i=1}^{n-1}\left(c_{i+1}-c_{i}\right) F_{t+k}^{E}\left(E_{t+k}^{c}+l_{i}\right)-c_{n} .
$$

Since $\left(c_{i+1}-c_{i}\right)>0$ and $F_{t+k}^{E}$ strictly increasing, we have $h^{\prime}\left(E_{t+k}^{c}\right)>0, \forall E_{t+k}^{c}$. It can then be concluded that the solution of $h\left(E_{t+k}^{c}\right)=0$ exists and is unique. This conclusion can be 
extended to the class of strictly convex loss functions such that $g^{\prime}(0)=0$, since $g$ could then be described by (18), with possibly $n$ tending toward infinity.

\section{ACKNOWLEDGMENT}

The authors would like to thank ESB National Grid and APX/ TenneT for providing the data, as well as three anonymous reviewers for their valuable comments.

\section{REFERENCES}

[1] L. H. Nielsen, et al., "Wind power and a liberalised North European electricity exchange," in Proc. Eur. Wind Energy Conf. (EWEC'99), Nice, France, Mar. 1999, pp. 379-382.

[2] K. Skytte, "The regulating power market on the Nordic power exchange Nord Pool: An econometric analysis," Energy Econ., vol. 21, pp. 295-308, 1999.

[3] A. H. Murphy, "What is a good forecast? An essay on the nature of goodness in weather forecasting," Weather Forecast., vol. 8, no. 2, pp. 281-293, 1993.

[4] H. Holttinen, "Optimal electricity market for wind power," Energy Policy, vol. 33, no. 16, pp. 2052-2063, Nov. 2004.

[5] J. Usaola, O. Ravelo, G. Gonzalez, F. Soto, C. Davila, and B. DiazGuerra, "Benefits for wind energy in electricity markets from using short term wind power prediction tools-A simulation study," Wind Eng., vol. 28, no. 1, pp. 119-128, Jan.-Feb. 2004.

[6] G. N. Bathurst, J. Weatherhill, and G. Strbac, "Trading wind generation in short-term energy markets," IEEE Trans. Power Syst., vol. 17, no. 3 , pp. 782-789, Aug. 2002.

[7] M. S. Roulston, D. T. Kaplan, J. Hardenberg, and L. A. Smith, "Using medium-range weather forecasts to improve the value of wind energy production," Renew. Energy, vol. 28, pp. 585-602, 2003.

[8] J. B. Bremnes, "Probabilistic wind power forecasts using local quantile regression," Wind Energy, vol. 7, no. 1, pp. 47-54, Jan.-Mar. 2004.

[9] U. Linnet, "Tools supporting wind energy trade in deregulated markets," Master's thesis, Technical Univ. Denmark, Kgs. Lyngby, Denmark, 2005, IMM-Thesis-2005-56.

[10] J. Matevosyan and L. Söder, "Minimization of imbalance cost trading wind power on the short-term power market," IEEE Trans. Power Syst., vol. 21, no. 3, pp. 1396-1404, Aug. 2006.

[11] R. Madlener and M. Kaufmann, Power Exchange Spot Market Trading in Europe: Theoretical Considerations and Empirical Evidence, OSCOGEN Project Deliverable Rep. 5.1b, Mar. 2002.

[12] J. Usaola and P. de Arriba Segurado, Wind Prediction in Electricity Markets, ANEMOS Project Deliverable Report D8.2, Nov. 2005. [Online]. Available: http://anemos.cma.fr.

[13] P. E. Morthorst, "Wind power and the conditions at a liberalized power market," Wind Energy, vol. 6, pp. 297-308, Jul.-Sep. 2003.

[14] B. B. Kristoffersen, B. Donslun, and P. B. Eriksen, "Impacts of largescale wind power on the power market," in Proc. 4th Int. Workshop Large-Scale International Wind Power Transmission Network Offshore Wind Farms, Billund, Denmark, Oct. 2003.

[15] G. N. Bathurst and G. Strbac, "Value of combining energy storage and wind in short-term energy and balancing markets," Elect. Power Syst. Res., vol. 67, pp. 1-8, 2003.

[16] E. D. Castronuovo and J. A. Peças Lopes, "On the optimization of the daily operation of a wind-hydro power plant," IEEE Trans. Power Syst., vol. 19, no. 3, pp. 1599-1606, Aug. 2004.

[17] G. Giebel, R. Brownsword, and G. Kariniotakis, State of the Art on Short-Term Wind Power Prediction, ANEMOS Project Deliverable Rep. D1.1, Jun. 2003. [Online]. Available: http://anemos.cma.fr.

[18] G. Kariniotakis and P. Pinson, "Uncertainty of short term wind power forecasts-A methodology for on-line assessment," in Proc. IEEE PMAPS'04 Conf., Ames, IA, Sep. 2004, pp. 729-736.

[19] P. Pinson, "Estimation of the uncertainty in wind power forecasting," Ph.D. dissertation, Ecole des Mines de Paris, Paris, France, 2006.
[20] S. Galloway, G. Bell, G. Burt, J. McDonald, and T. Siewierski, "Managing the risk of trading wind energy in a competitive market," Proc. Inst. Elect. Eng., Gen., Transm., Distrib., vol. 153, no. 1, pp. 106-114, Jan. 2006.

[21] M. Olsson and L. Söder, "Generation of regulating power price scenarios," in Proc. IEEE PMAPS'04 Conf., Ames, IA, Sep. 2004, pp. $26-31$.

[22] A. J. Conejo, J. Contreras, R. Espínola, and M. A. Plazas, "Forecasting electricity prices for a day-ahead pool-based electricity energy market," Int. J. Forecast., vol. 21, no. 3, pp. 435-462, 2005.

[23] Y.-M. Saint Drenant, Wind Power Predictions Analysis-Part 1: TenneT Imbalance Price System. Development of a Model for TenneT Imbalance Price System, Energy Research Center of the Netherlands, ECN report-ECN-I-02-010, 2002.

[24] V. Miranda and L. M. Proença, "Probabilistic choice vs. risk analysisconflicts and synthesis in power system planning," IEEE Trans. Power Syst., vol. 13, no. 3, pp. 1038-1043, Aug. 1998.

[25] M. Sewalt and C. de Jong, "Negative prices in electricity markets," Commodities Now Jun. 2003. [Online]. Available: http://www.commodities-now.com.

[26] A. Boogert and D. Y. Dupont, "On the effectiveness of the anti-gaming policy between the day-ahead and real-time electricity markets in the Netherlands," Energy Econ., vol. 27, no. 5, pp. 752-770, Sep.-Oct. 2005.

[27] G. Kariniotakis and D. Mayer, "An advanced on-line wind resource prediction system for the optimal management of wind parks," in Proc. IEEE MedPower'02 Conf., Athens, Greece, Nov. 2002.

[28] G. Kariniotakis, et al., "What performance can be expected by shortterm wind power prediction models depending on site characteristics?," in Proc. Eur. Wind Energy Conf. (EWEC'04), London, U.K., Nov. 2004.

[29] H. Madsen, P. Pinson, H. A. Nielsen, T. S. Nielsen, and G. Kariniotakis, "Standardizing the performance evaluation of short-term wind power prediction models," Wind Eng., vol. 29, no. 6, pp. 475-489, Dec. 2005.

Pierre Pinson was born in Poitiers, France. He received the M.Sc. degree in applied mathematics from the National Institute for Applied Sciences (INSA), Toulouse, France, in 2002 and the Ph.D. degree in energetic from Ecole des Mines de Paris, Paris, France, in 2006.

$\mathrm{He}$ is currently with the Informatics and Mathematical Modeling Department of the Technical University of Denmark, Lyngby, Denmark, as an Assistant Professor. His research interests include among others forecasting methods, uncertainty estimation, optimization under uncertainty, decision sciences, and renewable energies.

Christophe Chevallier was born in France in 1981. He received the M.Eng. degree in 2004 from EPF, Sceaux, France, and the M.Sc. degree in electrical engineering in 2005 from National Polytechnical Institute (INPT), Toulouse, France. He is currently pursuing the Ph.D. degree in energetic from Ecole des Mines de Paris, Paris, France.

He is currently with the Center for Energy and Processes of Ecole des Mines de Paris as a Research Engineer. His research interests include stochastic optimization and distributed generation planning.

George N. Kariniotakis (S'95-M'02) was born in Athens, Greece. He received the Engineering and M.Sc. degrees from the Technical University of Crete, Crete, Greece, in 1990 and 1992, respectively, and the Ph.D. degree from Ecole des Mines de Paris, Paris, France, in 1996.

$\mathrm{He}$ is currently with the Center for Energy and Processes of Ecole des Mines de Paris as a Senior Scientist. He is the Scientific and Technical Coordinator of the EU project Anemos. His research interests include among others renewable energies, wind power forecasting, distributed generation, and artificial intelligence. 